\title{
Mechanical and morphology properties of Poly(butylene succinate) reinforced by magnesium hydroxide sulfate hydrate whisker
}

\author{
Chuanhui Gao ${ }^{a}$, Zetian Li, Xinhua Zhang, Jing Wang, Yuetao Liu, Wenpeng Zhao and Juan Liu \\ College of Chemical Engineering, Qingdao University of Science \& Technology, Qingdao 266042, China
}

\begin{abstract}
Poly (butylene succinate) reinforced by magnesium hydroxide sulfate hydrate whisker was prepared. The mechanical properties, hardness and morphology of the composites were investigated. The tensile strength, flexural strength and flexural modulus were significantly increased with the adding of filler mass fraction, which about increased by $26.9 \%$, $97.0 \%$ and $187.6 \%$ corresponding to the addition amount of $25 \mathrm{wt} \%$ relative to neat PBS, respectively. On the contrary, the hardness of the composites was most reduced by $28.2 \%$. The elongation at break of PBS-5\%MHSH composites merely exhibited a slightly increased. The interfacial adhesion between whiskers and matrix was confirmed by scanning electron microscopic (SEM).
\end{abstract}

Keywords: Poly(butylene succinate); magnesium hydroxide sulfate hydrate; composites.

\section{Introduction}

For environmental friendly and biological materials, the preparation and application of Poly (butylene succinate) (PBS) based biodegradable materials is the emerging field of research [1,2]. PBS(number-average molecular weight and density are $5.25 \times 10^{4} \mathrm{~g} / \mathrm{mol}$ and $1.26 \mathrm{~g} / \mathrm{cm}^{3}$ ) is a new kind of biodegradable polymer materials, Which is synthesized from succinic acid and 1,4-butanediol in variety of grade to give response to different application, such as packaging, compost bags, agricultural films, coatings and textiles[3-6]. The polymer is semicrystalline, having good processability, favourable thermostability and physical properties that are similar to polyethylene [7-10]. It is said that PBS could undergo biodegradation during disposal in compost, moist and humus soil $[11,12]$. Commonly, in the natural environment, the biodegradable polymer will be decomposed by microorganisms to $\mathrm{CH}_{4}, \mathrm{CO}_{2}, \mathrm{H}_{2} \mathrm{O}$ and inorganic products under anaerobic or aerobic conditions [13].However, further for achieving an advantageous property such as tensile strength, flexural strength, flexural modulus and reduced the cost, thus it is modified by a variety of methods including reinforcement with inorganic whiskers[14-15].

Table 1. Properties of magnesium hydroxide sulfate hydrate whisker

\begin{tabular}{ccccc}
\hline Molecular formula & $\begin{array}{c}\text { Specific Gravity } \\
\left(\mathbf{g} / \mathbf{c m}^{3}\right)\end{array}$ & $\begin{array}{c}\text { Length } \\
(\boldsymbol{\mu m})\end{array}$ & $\begin{array}{c}\text { Diameter } \\
(\boldsymbol{\mu m})\end{array}$ & Aspect Ratio \\
\hline $5 \mathrm{Mg}(\mathrm{OH})_{2} \cdot \mathrm{MgSO}_{4} \cdot 3 \mathrm{H}_{2} \mathrm{O}$ & 2.3 & $10-60$ & $<1.0$ & $30-40$ \\
\hline
\end{tabular}

a Corresponding author : chuanhuigao@126.com 
Magnesium hydroxide sulfate hydrate whisker(The properties are given in table 1) (MHSH) were a single crystal fiber material with high stiffness and strength, which also have been proved to be well suited as additives for polymers such as resins and plastics, acting as fillers or reinforcers with the aim of enhancing the polymers' mechanical properties and thermal stability [16]. At the same time, owing to the proximity of expansion coefficient and their small diameter, whiskers are easy to disperse in the matrix materials so that the composites showed excellent physical and mechanical performance $[17,18]$. In addition, Magnesium hydroxide sulfate hydrate whiskers are different from other toxic whisker restricted for use in the materials, because it's non-toxic, small amount of smoke, good flame retardant and low cost[19,20]. As a consequence, MHSH are widely used for the preparation of composite material aimed to alter the corresponding performance of the composite materials, including mechanical properties, flame retardant performance and the thermal deformation temperature and so on[21]. However, PBS reinforced by MHSH has not been reported so far.

In this paper, the effect of the mass fraction on the mechanical properties of PBS/MHSH composites was investigated, including tensile strength, flexural strength, flexural modulus, the elongation at break and the hardness. Besides, the dispersion of MHSH in the matrix and the interfacial adhesion between the MHSH and the PBS were also observed.

\section{Experimental}

\subsection{The preparation of PBS/MHSH composites}

The pure PBS and composites containing 5, 10, 15, 20, 25wt\% MHSH were prepared in a Midi co-rotating twin screw extruder at $145^{\circ} \mathrm{C}$. The extrudates were directly injection into standard specimen (standard dog-bone tensile bars and flexural samples with dimensions of $75 \times 10 \times 4 \mathrm{~mm}^{3}$ ) and the mold temperature was maintained at $35^{\circ} \mathrm{C}$.

\subsection{Experimental procedures and characterizations}

Tensile tests for the composites were carried out according to GB/T1024.2-2006 using a Universal Testing Machine (AI-7000S, China) at a crosshead speed of $50 \mathrm{~mm} / \mathrm{min}$. The flexural measurements were carried out in accordance with GB/T934-2008 at a crosshead speed of $2 \mathrm{~mm} / \mathrm{min}$. Five measurements were done for each sample and final results were reported as averaged values. All of the tests were done at room temperature.

The hardness of pure PBS and its composites were characterized by Rockwell hardness tester (XHR-150, DONGHUA, China). The pressure head adopts the ordinary steel ball and according to the indentation to calculate the hardness. The testing values were dimensionless and HRB merely represented Rockwell scale.

Morphology of the composites was observed on SEM (JSM-6700F, JEOL, Japan) at an accelerating voltage of $12 \mathrm{kV}$ at high vacuum. All the samples were cry-fractured using liquid nitrogen and sputter-coated with gold prior to examination.

\section{Results and discussion}

\subsection{Tensile properties}

Fig 1(a) showed the role of different MHSH mass fraction on the tensile properties of PBS. Obviously, the tensile strength of PBS/MHSH composites with the increase of filler mass fraction was performed a trend of increase. When the filling amount was less than $10 \mathrm{wt} \%$, the tensile strength was not significantly promoted. However, the tensile strength compared with the pure PBS appeared significantly enhanced when the filling amount was more than $15 \mathrm{wt} \%$, especially while the addition 
amount of $25 \mathrm{wt} \%$, the tensile strength was $51.93 \mathrm{Mpa}$, which relative to pure matrix (40.93Mpa) was increased by $26.9 \%$. Similarly, Michael Snowdon [9] et al. filled 1, 3, and 5wt\% carbon black in PBS matrix and found the tensile stress at yield nearly remained constant, but there was no further increase the mass fraction of carbon black.

According to the scanning electron microscopy, it can be found that the structure of MHSH possessed of the larger aspect ratio and uniform length. Therefore, as the dispersed phase, whisker was evenly dispersed in the matrix of polyester, and the substrate material was the main part of the under external force when the whisker content was less than $10 \mathrm{wt} \%$. As a result, the tensile strength of the composite material corresponding to pure polyester had no obvious change. However, when whisker content exceeded $15 \mathrm{wt} \%$, the density dispersed in matrix materials was significantly increased though whisker was still served as the dispersed phase. Meanwhile, the whiskers gradually had the ability to withstand external force and can transfer and disperse stress. When the stress passed whisker from the polymeric matrix, shear stress generated in the interface was effectively added in the presence of high aspect ratio fibrous whiskers. As a consequence, the tensile strength was increased.

The elongation at break of the pure PBS and PBS/MHSH composites was demonstrated in Fig 1(b). It can be seen that the samples were first rose and then gone down latter with the increase of adding amount. Compared to the neat PBS, the composite with 5wt\% MHSH whiskers exhibited a slightly increased corresponding to others. The composites containing whiskers content less than $10 \mathrm{wt} \%$ showed ductile behavior with obvious necking during tension, so the elongation at break similar to the neat PBS surpass $150 \%$. However, the composites containing whiskers content more than $15 \mathrm{wt} \%$ exhibited yielding and showed brittle fracture so that the sample values appeared drastically declining. This was mainly because the whisker was a high-strength brittle material and its elongation was quite small. When the tensile stress was outstripped the range of composite materials could stand, the capacity of stress transmission between the whisker and matrix interface was destroyed. The tensile stress was far more than the endurance of the base material, caused rapid fracture in the composite materials.

\subsection{Flexural properties}

The Fig 1(c) showed that the flexural strength and flexural modulus of the PBS/MHSH composites both increased gradually with whiskers addition. More precisely, the flexural strength and flexural modulus of the composite were $42.63 \mathrm{Mpa}$ and $1651.62 \mathrm{Mpa}$ respectively, which were higher than pure polyester $(20.63 \mathrm{Mpa}, 574.35 \mathrm{Mpa})$ when the whiskers filled content were $25 \mathrm{wt} \%$. The increased range was about $97.0 \%$ and $187.6 \%$, respectively. The enhanced stiffness of the composites was attributed to the natural properties of the inorganic whiskers whose bending strength and bending modulus were greater than the matrix materials. It is also important to point out that the highest values were obtained for the composites in relation to whiskers dispersed in the matrix could resistant local strain and pass polyester to the surrounding whiskers, when the external force to the composites. 


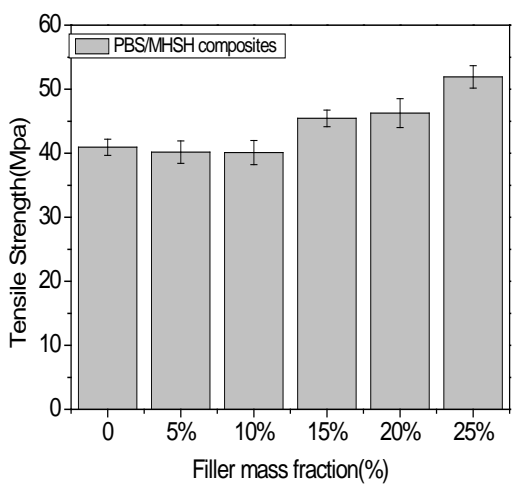

(a)

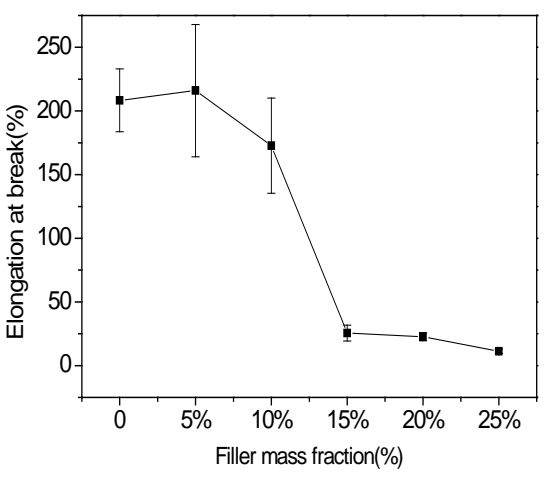

(b)

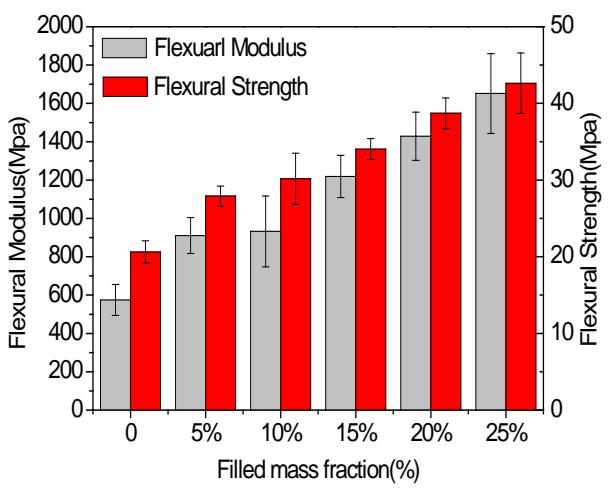

(c)

Figure 1. The mechanical properties of PBS/MHSH composites comprising different mass fraction: (a) tensile strength (b) elongation at break (c) flexural modulus and strength

\subsection{Hardness testing}

The effects of MHSH whiskers loading on the composites hardness were showed in Figure 2. In generally, the hardness of the composites should increase with in corporation of whiskers due to the inorganic materials with higher stiffness than the polyester. However, it was observed that the neat PBS exhibited better hardness over all the PBS/MHSH composites. It was noteworthy to mention that the hardness testing values gradually began to decline when the whisker content exceed $15 \mathrm{wt} \%$. This phenomenon was in good agreement with the elongation at break of the pure PBS and PBS/MHSH composites. The minimum value obtained for an amount of $25 \mathrm{wt} \%$ whiskers was 68.9 , which relative to the matrix material was most reduced by $28.2 \%$. It is also noticed that when the whisker content less than $10 \mathrm{wt} \%$, the hardness of the composites values with respect to the neat PBS was not significantly reduce or increase. It was because a phase separation, found in SEM photos (Fig 3), occurred between the whisker and matrix. It is evidenced by the presence of the gap appeared in the interface. The rockwell hardness tester was based on the indentation depth to calculate the hardness, therefore, the depth of the indentation increased under the great pressure. 


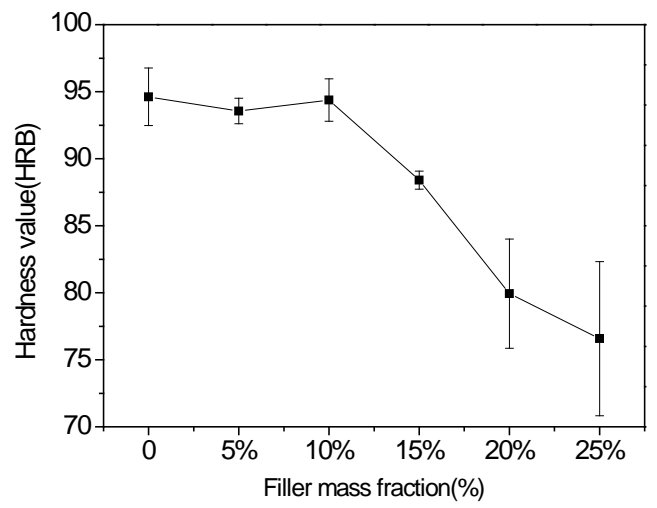

Figure 2. Rockwell hardness of pure PBS and PBS/MHSH composites comprising different mass fraction

\subsection{Scanning electron microscopy (SEM) micrograph}

With the aim of assessing the extent of filler dispersion within the polyester matrix, SEM images recorded over cry-fractured surfaces have been performed [22]. Fig 3 (a) showed an SEM micrograph of MHSH whiskers at low magnification. It was noticed that the whiskers exhibited fibrous appearance and a large aspect ratio owing to their small diameter. From (b) and (c) images, it was rather difficult to observe the whiskers in the polyester probably because the filler mass fraction was too lower to be covered and coated by the matrix. Compared to the $5 \mathrm{wt} \%$ and $10 \mathrm{wt} \%$, the exceeding $15 \mathrm{wt} \%$ composites where the whiskers can be seen readily among the PBS materials. It was noteworthy to stress that the MHSH whiskers resulted in some gaps and holes (marked by the black arrow) which can be clearly seen in picture (e) and (f) between matrix and filler. This predominantly elucidated that the presence of immiscible or incompatibility caused phase separation. There were also some whiskers bunching together and forming aggregates in certain areas.
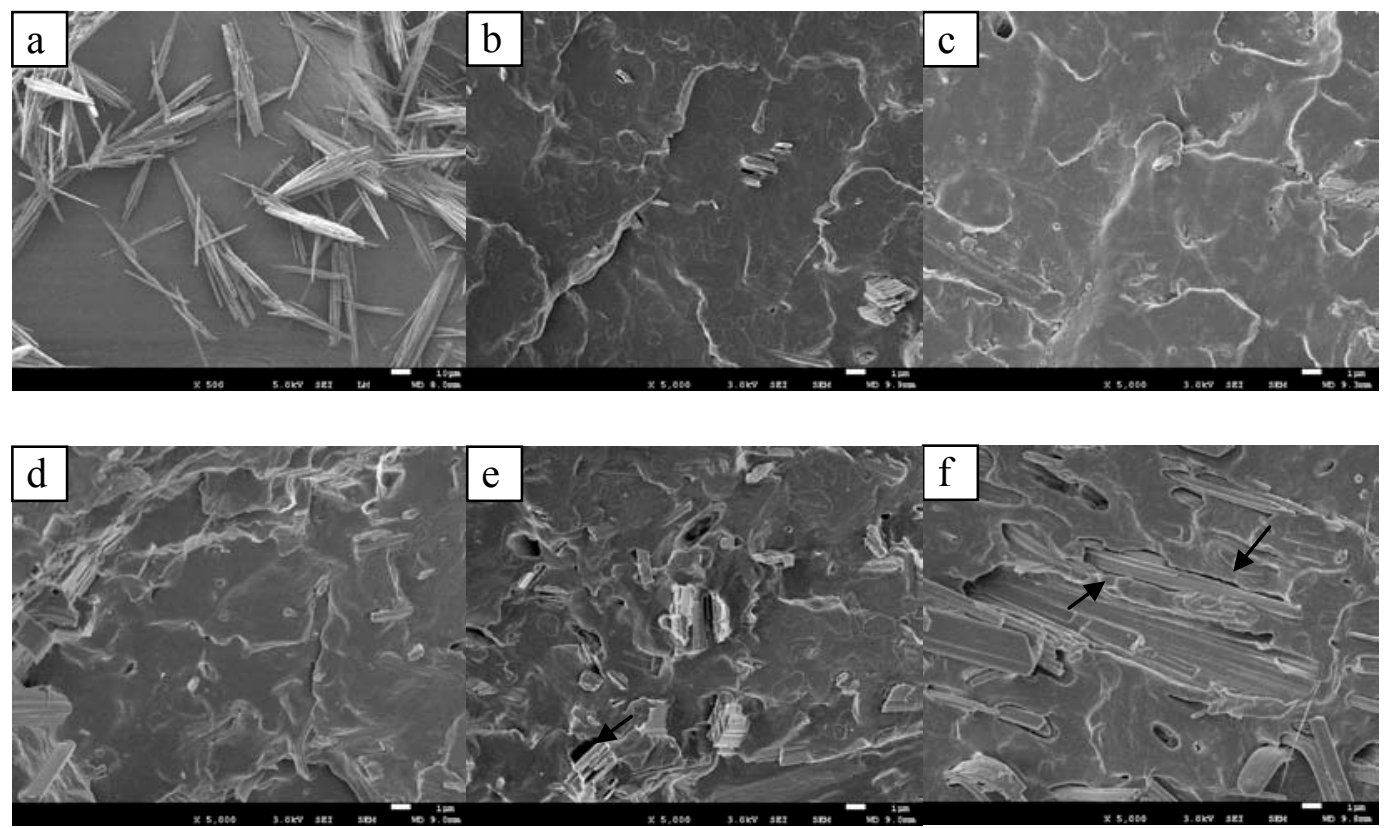

Figure 3. SEM images of the whiskers and the cry-fractured surfaces for PBS/MHSH composites: (a)MHSH whiskers, (b)PBS-5\%MHSH, (c)PBS-10\%MHSH, (d)PBS-15\%MHSH, (e)PBS-20\%MHSH, (f)PBS-25\%MHSH 


\section{Conclusion}

PBS composites filled by magnesium hydroxide sulfate hydrate whiskers were successfully prepared in a twin-screw extruder followed by injection molding. The composites mechanical properties demonstrated improvements in tensile, flexural and the elongation at break upon addition up to the $5 \mathrm{wt} \%$ MHSH loading. The composites containing lower whisker content $(<10 \mathrm{wt} \%)$ showed ductile fracture and exhibited necking behavior. The hardness values were gradually declined with the adding of filler mass fraction. The SEM images suggested whiskers were immiscible with the PBS matrix due to without surface modification.

\section{Acknowledgements}

This work is supported by the National Natural Science Foundation of China (51308314) and applied basic research projects of Qingdao (15-9-1-101-jch).

\section{References}

1. Platt D K. Biodegradable polymers: market report [M]. iSmithers Rapra Publishing. (2006)

2. Lee S M, Cho D, Park W H, et al. Novel silk/poly (butylene succinate) biocomposites: the effect of short fibre content on their mechanical and thermal properties [J]. Composites Science and Technology, 65(3): 647-657 (2005)

3. Liu L, Yu J, Cheng L, et al. Biodegradability of poly (butylene succinate) (PBS) composite reinforced with jute fibre[J]. Polymer Degradation and Stability, 94(1): 90-94. (2009)

4. Gao C H, Ding L, Wu Y M, et al. Low-Cost Synthesis and Morphology Control of Magnesium Oxysulfate Hydrate Whiskers[C]//Advanced Materials Research. 936: 986-991. (2014)

5. Liu B, Zhang Y, Wan C, et al. Thermal stability, flame retardancy and rheological behavior of ABS filled with magnesium hydroxide sulfate hydrate whisker [J]. Polymer Bulletin, 58(4): 747-755. (2007)

6. Lu H, Hu Y, Yang L, et al. Study of the fire performance of magnesium hydroxide sulfate hydrate whisker flame retardant polyethylene [J]. Macromolecular Materials and Engineering, 289(11): 984-989. (2004)

7. Tjong S C, Meng Y Z. Morphology and performance of potassium titanate whisker-reinforced polypropylene composites [J]. Journal of applied polymer science, 70(3): 431-439. (1998)

8. Tran T, Lee B H, Yang H S, et al. Use of starch granules melting to control the properties of bio-flour filled polypropylene and poly (butylene succinate) composites: Mechanical properties[J]. Starch-Stärke, 63(10): 637-648. (2011)

9. Snowdon M, Mohanty A K, Misra M. Melt Processing and Characterization of Bionanocomposites Made from Poly (butylene succinate) Bioplastic and Carbon Black [J]. Macromolecular Materials and Engineering, 300(1): 118-126. (2015)

10. Sinha Ray S, Okamoto K, Okamoto M. Structure-property relationship in biodegradable poly (butylene succinate)/layered silicate nanocomposites[J]. Macromolecules, 36(7): 2355-2367. (2003)

11. Fujimaki T. Processability and properties of aliphatic polyesters, 'BIONOLLE', synthesized by polycondensation reaction[J]. Polymer degradation and stability, 59(1): 209-214. (1998)

12. Kim H S, Kim H J, Lee J W, et al. Biodegradability of bio-flour filled biodegradable poly (butylene succinate) bio-composites in natural and compost soil[J]. Polymer Degradation and Stability, 91(5): 1117-1127. (2006)

13. Thirmizir M Z, Ishak Z A, Taib R M, et al. Kenaf-bast-fiber-filled biodegradable poly (butylene succinate) composites: Effects of fiber loading, fiber length, and maleated poly (butylene succinate) on the flexural and impact properties [J]. Journal of Applied Polymer Science, 122(5): 3055-3063. (2011)

14. Okamoto K, Sinha Ray S, Okamoto M. New poly (butylene succinate)/layered silicate 
nanocomposites. II. Effect of organically modified layered silicates on structure, properties, melt rheology, and biodegradability [J]. Journal of Polymer Science Part B: Polymer Physics, 41(24): 3160-3172. (2003)

15. Someya Y, Nakazato T, Teramoto N, et al. Thermal and mechanical properties of poly (butylene succinate) nanocomposites with various organo-modified montmorillonites [J]. Journal of applied polymer science, 91(3): 1463-1475. (2004)

16. Fang $\mathrm{S}, \mathrm{Hu} \mathrm{Y}$, Song $\mathrm{L}$, et al. Mechanical properties, fire performance and thermal stability of magnesium hydroxide sulfate hydrate whiskers flame retardant silicone rubber [J]. Journal of Materials Science, 43(3): 1057-1062. (2008)

17. $\mathrm{Lu} \mathrm{H}, \mathrm{Hu} \mathrm{Y}$, Xiao J, et al. Magnesium hydroxide sulfate hydrate whisker flame retardant polyethylene/montmorillonite nanocomposites[J]. Journal of materials science, 41(2): 363-367. (2006)

18. Ding Y, Zhang G, Zhang S, et al. Preparation and characterization of magnesium hydroxide sulfate hydrate whiskers [J]. Chemistry of materials, 12(10): 2845-2852. (2000)

19. Zhou Z, Sun Q, Hu Z, et al. Nanobelt formation of magnesium hydroxide sulfate hydrate via a soft chemistry process [J]. The Journal of Physical Chemistry B, 110(27): 13387-13392. (2006)

20. Keefer K D, Hochella M F, de Jong B H W S. The structure of the magnesium hydroxide sulfate hydrate MgSO4. $1 / 3 \mathrm{Mg}(\mathrm{OH}) 2.1 / 3 \mathrm{H} 2 \mathrm{O}[\mathrm{J}]$. Acta Crystallographica Section B: Structural Crystallography and Crystal Chemistry, 37(5): 1003-1006. (1981)

21. Ding Y, Zhang G, Wu H, et al. Nanoscale magnesium hydroxide and magnesium oxide powders: control over size, shape, and structure via hydrothermal synthesis [J]. Chemistry of Materials, 13(2): 435-440. (2001)

22. Han S O, Son W K, Youk J H, et al. Electrospinning of ultrafine cellulose fibers and fabrication of poly (butylene succinate) biocomposites reinforced by them[J]. Journal of applied polymer science, 107(3): 1954-1959. (2008) 